\title{
COMMENTARY
}

\section{Delivering on the promise of biosimilars}

\author{
Arnold G. Vulto ${ }^{1,2}$ \\ 1 Clinical Pharmacology and Pharmacotherapy, KU Leuven, Leuven, Belgium \\ 2 Hospital Pharmacy, ErasmusMC, Rotterdam, the Netherlands \\ a.vulto@gmail.com
}

\begin{abstract}
Fifteen years of experience with biosimilar evaluation in Europe and advancement in the science behind biological medicines, provides a timely moment to open up debate as to whether the requirements for biosimilar approval could be further tailored. Further optimizing of data requirements to truly decisional information will allow to continuously deliver on the promise of biosimilars, providing benefits for patients and society.
\end{abstract}

\section{The promise of biosimilars}

The introduction of new, often expensive, medicines is placing increasing pressure on healthcare systems globally. As a result, healthcare systems around the world are faced with growing affordability issues. The market entry of generic medicines has been successful by resulting in significant cost savings, creating budgetary headroom for new medicines that enter the market. With the increasing loss of exclusivity rights for biological medicines, the market has opened up for similar versions of these products, biosimilars. In a similar way to the impact of generic medicines on the small molecules' market, biosimilars have the opportunity to generate competition in the biological medicines' segment, reducing treatment costs and increasing patient access.

The promise of biosimilars is all about cost and access. Price reductions have been reported to be as high as $60-80 \%$ of the originator list price (country and product dependent) $\left.{ }^{1}\right]$, and the introduction of biosimilars has been projected to result in savings of more than $€ 10$ billion between 2016 and 2020 in the EU5 countries (France, Germany, Italy, Spain and the UK) alone. $\left[^{2}\right]$ While leading to cost advantages for patients and payers, biosimilars provide the same quality, efficacy and safety as originator biological medicines. The benefit of the use of biosimilars extends beyond cost reduction, as the introduction of market competition makes biological medicines affordable for more patients. Biosimilar market entry has been shown to improve patient access to biological medicines (increases in the number of patients and/or earlier access to therapy). $\left[{ }^{2}\right]$ To deliver the promise of biosimilars, a solid regulatory framework, in combination with a competitive and long-term sustainable off-patent biological medicines market environment, is needed, which allows for multiple players on the market.

\section{The regulatory landscape}

Due to the natural variability, inherent to all biological medicines, and the manufacturing process, which is unique to each manufacturer, biological medicines cannot be identically copied. Regulators in the EU (European Medicines Agency, EMA) and the US (U.S. Food \& Drug Administration, FDA) have designed a pathway to assess the similarity of these similar 
versions of the originator biological medicines. Biosimilars are highly similar versions of an originator biological medicine (the reference product) with equal quality, efficacy and safety in patients. The EU pioneered the regulation of biosimilars, by introducing a legal framework for the approval of biosimilars in 2004. Since the first biosimilar approval in Europe in 2006 (Omnitrope®, a somatropin biosimilar), European regulators have gathered 15 years of experience in assessing biosimilars, approving 55 biosimilars for 15 distinct biological molecules. (See Wolff-Holz et al. 2019, table 1). ${ }^{3,4}$ The US implemented its abbreviated licensing pathway for biosimilars with the enactment of the Biologics Price Competition and Innovation Act (BPCIA) in 2010. The FDA approved its first biosimilar in March 2015 (Zarxio ${ }^{\circledR}$, a filgrastim biosimilar), almost ten years after the first biosimilar approval in Europe. To date, the FDA has approved 23 biosimilars $^{5}$, but only a handful are available on the market for patients. ${ }^{6}$

Patients and prescribers expect all medicines that are licensed to have a positive benefit-risk balance. When it comes to biosimilars, a positive benefit-risk balance is based on demonstrating biosimilarity, i.e. the quality, efficacy and safety of the biosimilar should equal those of the originator. There may be slight differences compared with the reference product, but the biosimilar needs to demonstrate that these minor differences are not clinically meaningful, i.e. have no impact on efficacy or safety. Biosimilars are evaluated based on a scientifically tailored dossier, consisting of extensive comparability studies between the biosimilar and its reference product.

\section{How biosimilars are being assessed: the biosimilarity exercise}

The origin of the biosimilarity exercise can be brought back to the experience regulators have built up by evaluating the pre- versus post-similarity of already-licensed biological medicines that undergo changes to their manufacturing process over the product lifecycle. ${ }^{5}$ Comparability is a well-established scientific principle that has been used for decades to evaluate product consistency before and after such a manufacturing change. Based on the experience of assessing comparability after a manufacturing change, the EMA and FDA designed a pathway applying the same scientific principles to assess the similarity of a biosimilar to its reference product. ${ }^{7}$

The extent of studies required to demonstrate comparability in the context of a manufacturing change depends on its expected impact on quality, safety and efficacy. Mostly, analytical and functional data are deemed sufficient. In general, for biosimilars, a larger scale of comparison is needed, including clinical data, as a biosimilar is developed by a different manufacturer using a newly developed cell line and its own manufacturing process. By demonstrating high similarity, the biosimilar can, however, largely rely on experience gained with the reference product, eliminating the need to repeat the entire toxicological and clinical development of the reference product.

The main goal of the biosimilarity exercise is to investigate potential product-related differences between the biosimilar and the reference product. Studies need to be tailored to the goal of the similarity exercise, i.e. studies need to be sensitive enough to detect and rule out potential product-related differences. The assessment is predominantly based on chemical, physical and pre-clinical pharmacological techniques, followed by a pharmacokinetic (PK) similarity assessment in humans. To address remaining residual uncertainty, one confirmatory randomized controlled trial in patients, to confirm similar efficacy, safety and immunogenicity, is generally requested. Finally, biosimilars are evaluated on the totality of the evidence gathered to demonstrate similarity to the reference product. Furthermore, biosimilars are subject to stringent post-marketing surveillance, as is the case for all biological medicines. 
As expected, the approach with the introduction of this new regulatory framework for biosimilars was set out to be very cautious, and now, after 15 years of experience with biosimilar evaluation in Europe and advancement in the science behind biological medicines, questions are increasingly being voiced as to whether the requirements for biosimilar approval could be further tailored. In fact, that question is the topic of two articles in this issue of BioDrugs (Wolff-Holz et al. 2019³ ; Webster et al. 2019²).

\section{A review of 15 years of EMA experience with biosimilars: past, present and future}

A team of European regulators reviewed the available guidance and European Public Assessment Reports (EPARs) for all biosimilars approved in the EU via the centralised procedure, in an effort to further identify the hierarchy in importance between different elements of the similarity exercise in establishing biosimilarity. ${ }^{3}$ The review provides extensive insight into the current thinking of regulators, highlighting how the totality of the evidence for biosimilarity has been weighted, taking the different components of the similarity exercise into account and discussing their impact on the overall similarity decision.

In addition to confirming the analytical and functional comparison as the foundation for biosimilarity, their analysis demonstrates that PK similarity is an essential similarity checkpoint for any biosimilar approval. As PK studies are in general more sensitive to detect potential product-related differences - the essential goal of the biosimilarity exercise - than clinical efficacy trials, evidence generated by PK studies is found to trump the results of the phase III efficacy trial. They argue that positive phase III trial results cannot overrule differences in PK assessment: there was not a single case where a phase III trial was decisive for the final decision taken by the regulators. As clinical trials are often insensitive to detect product-related differences, due to a variety of confounding patient- or disease-related factors, European regulators acknowledge that alternative approaches could be explored and are welcome to be discussed during scientific advice. They also argue that unnecessary duplication of clinical trials is unethical, costly and time-consuming (resulting in delayed availability of the biosimilar). Reducing clinical data requirements is already an accepted principle for biosimilars of less complex originator biological medicines, such as filgrastim and insulin. The question that remains is: when will enough experience and scientific advancement have been gathered to approve monoclonal antibody biosimilars without a large phase III comparative efficacy confirmatory clinical trial?

\section{A bold proposal to simplify and unify procedures and regulatory requirements for biosimilars}

In a second paper in this issue of BioDrugs, Webster, Wong and Woollett ${ }^{8}$ take this a step further with a bold proposal to rethink current approaches. These authors have written repeatedly - from a US perspective - about redundant work that was required by the FDA and that did not serve a scientific purpose. They see it as a fundamental flaw that the current development paradigm for biosimilars required by regulators in highly-regulated jurisdictions is derived from the development of originator drugs, which they see as unnecessarily burdensome and inefficient. The development paradigm for biosimilars structured by regulators takes insufficient account of pre-existing knowledge of the biosimilar and its reference product and the different opportunities for the use of inference in evaluating biosimilars and originator medicines. A foundational precept of the comparability of biological medicines is the predictability of the relationship between structure and function; two biological medicines that are highly alike analytically will almost inevitably have the same functionalities. Moreover, the sensitivity and specificity of modern analytical methods may 
discover appreciable analytical differences between biological medicines without detectable differences in their functionalities. ${ }^{9}$ It is worth noting the discrepancies that the authors show in table 1 between products approved in Europe and the US, which cannot be explained by differences in scientific data. They make two important observations, in agreement with Wolff-Holz et al. 2019. Firstly, not a single rejection of approval has been caused by a finding of lack of clinical equivalence between the biosimilar candidate and its reference product when the two products have been found earlier to be highly similar in analytical and human PK studies. Secondly, no biosimilar that was found to be highly similar to its reference product by both analytical and human PK studies has ever failed to be approved because it was found not to be clinically equivalent to its reference product in a powered study. It can therefore be argued that powered efficacy studies used as a confirmatory step for similar efficacy, do not necessarily provide decisional information.

Authors explain that the establishment of biosimilarity involves the discovery and evaluation of compositional differences between the biosimilar candidate and reference product with regard to their impact upon the clinical performance of the medicine. A focus on analytical comparability, and complementary methods that examine directly the quantitative biological effects of differences in composition (such as PKs), is more sensitive, precise, and efficient than the more diffuse information that results from statistical manipulation delivered by comparative efficacy studies.

Only those development studies necessary to enable a conclusion of biosimilarity would be justified, and should be required. The authors propose that a paradigm focused upon analytical resemblance - 'confirmation of sufficient likeness (CSL)' - should supersede the totality of the evidence paradigm for biosimilar development. The CSL concept is further defined in the opinion paper. As a scientific matter, the data presented in their opinion paper support the conclusion that CSL does not represent any reduction in the effective rigor with which biosimilars are evaluated, and will not change the nature of the product finally approved. Its implementation will be important in stimulating the availability of biosimilars globally.

In addition, the authors also propose a more intense collaboration between regulatory agencies to avoid the circularity and duplication that is inherent under the current arrangements of individual jurisdiction (in particular for the FDA and EMA). They call upon the societal responsibility of regulators to rethink the current system, which they argue is driving up development costs and causing delays in access. Or, as they say; "The societal importance of biosimilars is hard to overstate". Just to mention a single example of the impact of market competition: US citizens are paying around US $\$ 3000$ for a single dose (40mg) of Humira ${ }^{\circledR}$ (adalimumab originator product), while in Europe, due to competition induced by high-quality biosimilars, treatment cost has gone down to less than $10 \%$ of what US patients pay. ${ }^{10,11}$ This shows the impact of a different political and market climate on either side of the Atlantic Ocean.

\section{The outlook}

Both articles discussed in this editorial support the concept that adjustment of the regulatory requirements is justifiable, based on solid scientific facts (such as advancement in analytical technology and insight into structure-activity relationships) and based on the extensive experience that has accumulated, mainly in Europe, over some 15 years. With advancing knowledge and experience since 2004, it is time to rethink the level of clinical evidence needed to demonstrate biosimilarity, to reshape regulatory requirements and work towards further alignment between regulatory agencies. For the different approved biosimilars, switching trials have been performed and reviewed (Cohen HP et al, 2018 ${ }^{12}$; Barbier L et al, 
$2018^{13}$ ) and in over 170 studies describing clinical switching outcomes, no signals of underperformance or safety issues upon switching to the biosimilar are evident. Also, pharmacovigilance systems have not detected any alarming signals. So, it seems a timely moment for regulators to continue optimizing data requirements to those factors that matter and to refrain from demanding data that are costly and time consuming to generate and have little added value. This step can only be taken if educational efforts aimed at prescribers, pharmacists and patients are intensified. It will be a challenge for these stakeholders to understand that clinical trials for biosimilars are not always fit for purpose, as the decisional value these add to the science supporting biosimilarity can be limited. In addition, the scientific advice from regulators to sponsors should be timely in this respect, as design and execution of a clinical trial may take two years to complete.

\section{Conclusion}

In conclusion, both Wolff-Holz et al.[3] and Webster et al. [8] state that science has advanced in such a way that further tailoring of biosimilar development with respect to clinical scientific data can be explored. Webster et al.,[8] in addition, make a plea for more global unification and collaboration in assessment. These suggestions, to optimize licensing requirements and to unify assessment among regulators (reducing associated development costs), are welcome for all countries that are struggling with ever-increasing drug expenditures and treatment access, as this may incentivize multiple players to invest in biosimilar development, stimulating the availability of biosimilars and their positive effect on market competition globally. Further, these advances would allow a reduction of unnecessary waste of resources and clinical trials. Fifteen years of positive regulatory experience with biosimilars in Europe provides a good momentum to open up the debate towards further optimizing the balance between the regulatory "first; do no harm" principle and focussing licensing requirements to truly decisional information. Biosimilars are the single most effective way to drive down drug expenditure for biological medicines and advances in regulatory requirements can help in providing benefits for patients and society.

\section{Compliance with Ethical Standards}

\section{Funding}

No funding relevant to this commentary.

\section{Conflict of interest}

AGV is one of the founders of the Research Fund on Market Analysis of Biologics and Biosimilars following Loss of Exclusivity (MABEL Fund) and is involved in consulting, advisory work and speaking engagements for a number of companies, i.e., AbbVie, Accord, Amgen, Biogen, EGA, Pfizer/Hospira, Mundipharma, Roche, Novartis, Sandoz, and Boehringer Ingelheim.

\section{Acknowledgments}

AGV would like to thank Liese Barbier for the stimulating discussions and Michelle Derbyshire for linguistic review. 


\section{References}

1 IQVIA. The Impact of Biosimilar Competition in Europe. Quintiles IMS 2018; : 33.

2 IMS. Delivering on the Potential of Biosimilar Medicines: The Role of Functioning Competitive Markets Introduction. IMS Inst Healthc Informatics 2016; : 40.

3 Wolff-Holz E, Tiitso K, Vleminckx C, Weise M. Evolution of the EU Biosimilar Framework: past and future. BioDrugs 2019. doi:https://doi.org/10.1007/s40259-01900377-y.

$4 \quad$ European Medicines Agency. Biosimilar Medicines.

https://www.ema.europa.eu/en/medicines/field_ema_web_categories\%253Aname_fiel d/Human/ema_group_types/ema_medicine/field_ema_med_status/authorised-

36/ema_medicine_types/field_ema_med_biosimilar/search_api_aggregation_ema_me dicine_types/field_ema_med_biosim (accessed 9 Sep2019).

$5 \quad$ European Medicines Agency. ICH Topic Q 5 E Comparability of Biotechnological/Biological Products. 2005.

6 Harston A. FDA's Biosimilar Approvals Accelerate in 2018: How the U.S. Compares to Europe on Biosimilar Approvals and Products In the Pipeline.

2019.https://www.biosimilarsip.com/2019/02/05/fdas-biosimilar-approvals-acceleratein-2018-how-the-u-s-compares-to-europe-on-biosimilar-approvals-and-products-in-thepipeline/ (accessed 13 Sep2019).

7 Weise M, Bielsky MC, De Smet K, Ehmann F, Ekman N, Giezen TJ et al. Biosimilars: What clinicians should know. Blood 2012; 120: 5111-5117.

8 Webster CJ, Wong AC, Woollett GR. An Efficient Development Paradigm for Biosimilars. BioDrugs 2019. doi:10.1007/s40259-019-00371-4.

9 Planinc A, Dejaegher B, Vander Heyden Y, Viaene J, Van Praet S, Rappez F et al. Batch-to-batch N-glycosylation study of infliximab, trastuzumab and bevacizumab, and stability study of bevacizumab. Eur J Hosp Pharm 2017; 24: 286-292.

10 Eric Sagonowsky. AbbVie's massive Humira discounts are stifling Netherlands biosimilars: report | FiercePharma.

2019.https://www.fiercepharma.com/pharma/abbvie-stifling-humira-biosimcompetition-massive-discounting-dutch-report (accessed 13 Sep2019).

11 Sy Mukherjee. Protect at All Costs: How the Maker of the World's Bestselling Drug Keeps Prices Sky-High | Fortune. 2019.https://fortune.com/longform/abbvie-humiradrug-costs-innovation/ (accessed 13 Sep2019).

12 Cohen HP, Blauvelt A, Rifkin RM, Danese S, Gokhale SB, Woollett G et al. Switching Reference Medicines to Biosimilars: A Systematic Literature Review of Clinical Outcomes. Drugs 2018; 78: 463-478.

13 Barbier L., Ebbers H., Simoens S., Declerck P., Vulto A. HI. The safety of switching between reference biopharmaceuticals and biosimilars: a systematic review. VALUE Heal 2018; 21. doi:10.1016/j.jval.2018.09.1840. 